PROCEEDINGS OF THE

AMERICAN MATHEMATICAL SOCIETY

Volume 131, Number 4, Pages 1081-1091

S 0002-9939(02)06767-9

Article electronically published on September 19, 2002

\title{
THE SHORT RESOLUTION OF A LATTICE IDEAL
}

\author{
PILAR PISÓN CASARES
}

(Communicated by Wolmer V. Vasconcelos)

\begin{abstract}
The short resolution of a lattice ideal is a free resolution over a polynomial ring whose number of variables is the number of extremal rays in the associated cone. A combinatorial description of this resolution is given. In the homogeneous case, the regularity can be computed from this resolution.
\end{abstract}

\section{INTRODUCTION}

Let $I$ be a lattice ideal in $k\left[X_{1}, \ldots, X_{n}\right]=k[\mathbf{X}]$, where $k$ is a (commutative) field. The minimal free resolution of $I$ as a $k[\mathbf{X}]$-module has been studied by many authors. Recently combinatorial descriptions of this resolution have been given (see for example [2, [5] and references therein). In this paper we consider the minimal free resolution of $I$, not over $k[\mathbf{X}]$ but over a polynomial ring over $k$ whose number of variables is the number of extremal rays in the associated cone. This resolution is called the short resolution to distinguish it from the usual minimal free resolution, the long resolution.

Let $\Lambda$ be a generating set of the semigroup which parametrizes the associated algebraic variety. As in [6] we consider a partition of $\Lambda=E \cup A$, where $E$ consists of a chosen generator from each extremal ray. From $E$ we can define the "Apéry set" associated with the lattice ideal (see Definition 1.1). The terminology "Apéry" comes from the case of numerical semigroups 1 .

In section 1, Lemma 1.2 provides a way for computing the Apéry set using Gröbner Bases. The first step of the short resolution can be constructed from the Apéry set. The second step is described in Proposition 1.4 Now, the complete short resolution can be obtained by the usual methods (for example the Schreyer Theorem and its improvements [11]).

In section 2 a combinatorial description of the short resolution is given by means of simplicial complexes. This description is similar to the one which has been used in 7] (see also [12]) for the long resolution. As a corollary the main result (Corollary 2.2) of the paper is stated: The regularity of a homogeneous lattice ideal can be obtained from the short resolution. Curiously, in the case of toric curves, the classical techniques of Gruson, Lazarsfeld and Peskine 8 to study the regularity amount to understanding the short resolution. The cohomological machinery in 8

Received by the editors July 26, 2001 and, in revised form, November 21, 2001.

2000 Mathematics Subject Classification. Primary 13D02, 14M25; Secondary 13P10, 68W30.

Key words and phrases. Minimal free resolutions, simplicial complex, syzygy, lattice ideal, regularity.

This work was supported by MCyT Spain, BFM2000-1523.

(C)2002 American Mathematical Society 
is used by L'vovsky in [9] to give an explicit bound for the conductor of a numerical semigroup.

\section{APÉRY SETS}

Let $S$ be a cancellative commutative semigroup, with zero element and generated by $n$ elements $\Lambda=\left\{m_{1}, \ldots, m_{n}\right\}$. Thus, $S$ is a subsemigroup of a finitely generated abelian group. Denote $G(S)$ the smallest group containing $S$. The semigroup $k$ algebra is $k[S]=\bigoplus_{m \in S} k \chi^{m}\left(\chi^{m} \cdot \chi^{m^{\prime}}=\chi^{m+m^{\prime}}\right)$. The ideal of $S$ relative to $\Lambda$ is $\operatorname{ker}\left(\varphi_{0}\right)$, where $\varphi_{0}$ is the $k$-algebra morphism

$$
\varphi_{0}: k[\mathbf{X}] \longrightarrow k[S]
$$

defined by $\varphi_{0}\left(X_{i}\right)=\chi^{m_{i}}$. Notice that $\varphi_{0}$ is surjective, and hence $k[S] \simeq k[\mathbf{X}] /$ $\operatorname{ker}\left(\varphi_{0}\right)$.

Let $I$ be the ideal relative to a fixed $\Lambda$. Equivalently ([13), $I$ is a lattice ideal.

Assume that $S \cap(-S)=(0)$. Consider $k[S]$ with the natural $S$-grading and $k[\mathbf{X}]$ as an $S$-graded ring, assigning degree $m_{i}$ to $X_{i}$. Notice that $I$ is $S$-graded because $\varphi_{0}$ is an $S$-graded morphism of degree zero. The condition $S \cap(-S)=(0)$ says that $k[\mathbf{X}]_{m}$, the homogeneous elements of degree $m \in S$ in $k[\mathbf{X}]$, is a $k$-vector space of finite dimension (see [3]).

Assume that $\operatorname{rank}(G(S))=d$, let $V=G(S) \otimes \mathbf{z} \mathbf{Q}$, and let $C(S)$ be the cone generated by the image $\bar{S}$ of $S$ in $V$. The cone $C(S)$ is strongly convex because $S \cap(-S)=(0)$. Thus, if $f$ is the number of extremal rays of $C(S)$, then $f \geq d$. This implies that there exists a set $E \subset \Lambda$ with $\sharp E=f$, such that $C(E)=C(S)$, where $C(E)$ is the cone in $V(S)$ generated by $E$. Fix such a set $E$ and $A=: \Lambda \backslash E, \sharp A=$ $n-f=r$.

Definition 1.1. The Apéry set $Q$ of $S$ relative to $E$ is defined as

$$
Q=\{q \in S \mid q-e \notin S, \forall e \in E\} .
$$

Denote $k[E]$ as the subalgebra of $k[S]$,

$$
k[E]=\bigoplus_{m \in S_{E}} k \chi^{m},
$$

where $S_{E}$ is the subsemigroup of $S$ generated by $E$. Let $k\left[\mathbf{X}_{E}\right]$ be the polynomial ring in the $f$ indeterminates associated with $E . k\left[\mathbf{X}_{E}\right]$ can be projected onto $k[E]$; it is enough to associate to the indeterminate $X_{i}$ the symbol $\chi^{m_{i}}$, for any $m_{i} \in E$.

$k[S]$ is a $k[E]$-module, and therefore also a $k\left[\mathbf{X}_{E}\right]$-module. The set

$$
\left\{\chi^{q} \mid q \in Q\right\}
$$

is a minimal system of generators of $k[S]$ as $k[E]$-module, and therefore, also as a $k\left[\mathbf{X}_{E}\right]$-module. Since $k\left[\mathbf{X}_{E}\right]$ is noetherian, $Q$ is a finite set.

Assume, for the sake of simplicity, that

$$
E=\left\{m_{1}, \ldots, m_{f}\right\} \text { and } A=\left\{m_{f+1}, \ldots, m_{n}\right\} .
$$

Fix a total order on the monomials of $k[\mathbf{X}]=k\left[\mathbf{X}_{E}, \mathbf{X}_{A}\right], X_{1}<X_{2}<\cdots<X_{n}$, such that:

(1) $\mathbf{X}^{\alpha}<\mathbf{X}^{\beta}$ implies $\mathbf{X}^{\alpha+\gamma}<\mathbf{X}^{\beta+\gamma}$, for any $\alpha, \beta$ and $\gamma$.

(2) If $f=\sum a_{\alpha} \mathbf{X}^{\alpha} \in k[\mathbf{X}]$ has the leading monomial $\mathbf{X}^{\beta} \notin k\left[\mathbf{X}_{A}\right]$, then $\mathbf{X}^{\alpha} \notin$ $k\left[\mathbf{X}_{A}\right]$, for any $\alpha$ with $a_{\alpha} \neq 0$. 
For example, we can consider the lex - inf order which is defined

$$
\alpha>_{\text {lex-inf }} \beta \Longleftrightarrow \alpha<_{\text {lex }} \beta,
$$

where the lex order is the lexicographic order for $X_{1}>\cdots>X_{n}$.

Every order with these properties is not a well-ordering. However, since there exists only a finite number of monomials of $S$-degree $m \in S$, a Gröbner basis of $I$ can be computed from any $S$-graded generating set of $I$. Assume that $\Gamma$ is the reduced Gröbner basis of $I$ for such an order. Let $\mathcal{B}$ be the set of monomials $\mathbf{X}_{A}^{\alpha}$ which are not divisible by any leading monomial of $\Gamma$.

\section{Lemma 1.2.}

$$
Q=\left\{m \in S \mid m=\sum_{i=f+1}^{n} \alpha_{i} m_{i}, \text { where } \mathbf{X}_{A}^{\alpha} \in \mathcal{B}\right\},
$$

and in particular, $\mathcal{B}$ is finite.

Proof. We will use the Hironaka division remainder of a monomial by a binomial, a monomial. Moreover, if a monomial $\mathbf{X}^{\alpha}$ of $S$-degree $m=\sum_{i=1}^{n} \alpha_{i} m_{i}$ is divided by the reduced Gröbner basis, $\Gamma$, the remainder $\mathbf{X}^{\beta}$ is also of degree $m$, i.e. $m=$ $\sum_{i=1}^{n} \beta_{i} m_{i}$. Thus, if $\alpha \neq \beta$, we obtain a new writing of $m$ in the function of the generators of $S$.

Let $\mathbf{X}_{A}^{\alpha}=X_{f+1}^{\alpha_{f+1}} \cdots X_{n}^{\alpha_{n}} \in \mathcal{B}$, and let $m=\sum_{i=f+1}^{n} \alpha_{i} m_{i}$. If $m$ does not admit another way of writing in function of the generators, then $m \in Q$ and we are done. Otherwise, $m=\sum_{i=1}^{n} \beta_{i} m_{i}$ for some $\beta_{i} \in \mathbf{N}$, and $\mathbf{X}_{A}^{\alpha}-\mathbf{X}^{\beta} \in I$. The remainder of $\mathbf{X}_{A}^{\alpha}-\mathbf{X}^{\beta}$ by $\Gamma$ is zero. Thus, $\mathbf{X}^{\beta}$ is divisible by some leading monomial of $\Gamma$. Therefore, $\mathbf{X}_{A}^{\alpha}$ is divisible by some non-leading monomial of $\Gamma$, which will only have variables corresponding to $A$. Property 2 of the order guarantees that $\mathbf{X}^{\beta} \in k\left[\mathbf{X}_{A}\right]$. Thus, $m \in Q$.

Reciprocally, let $m \in Q$. It is possible to write $m=\sum_{i=f+1}^{n} \alpha_{i} m_{i}$, for some $\alpha_{i} \in \mathbf{N}$. Suppose that $\mathbf{X}_{A}^{\alpha}$ is divisible by some leading monomial of $\Gamma$. Thus, the remainder of $\mathbf{X}_{A}^{\alpha}$ by $\Gamma$ is $\mathbf{X}^{\beta}$, which is not divisible by any leading monomial of $\Gamma$ and it is of degree $m$. Since $m \in Q, \mathbf{X}^{\beta}=\mathbf{X}_{A}^{\beta} \in \mathcal{B}$ and we are done.

$\mathcal{B}$ is finite because $Q$ is finite and any element in $S$ only admits a finite number of writings in the function of the generators.

Let $l_{0}$ be the cardinality of $\mathcal{B}=\left\{\mathbf{X}_{A}^{\alpha_{1}}, \ldots, \mathbf{X}_{A}^{\alpha_{l_{0}}}\right\}$, and define the $k\left[\mathbf{X}_{E}\right]$-module morphism

$$
\Psi_{0}: k\left[\mathbf{X}_{E}\right]^{l_{0}} \longrightarrow k[S],
$$

$\Psi_{0}\left(e_{i}\right)=\mathbf{X}_{A}^{\alpha_{i}}+I$, where we are using the isomorphism $k[S] \simeq k[\mathbf{X}] / I$. Equivalently, $\Psi_{0}\left(e_{i}\right)=\chi^{q_{i}}$, where $q_{i} \in Q$ is the $S$-degree of the binomial $\mathbf{X}_{A}^{\alpha_{i}}$. (It is possible that the cardinality of $\mathcal{B}$ is greater than that of $Q$, and therefore $q_{i}=q_{j}$, for some $i \neq j$.)

$\Psi_{0}$ is surjective because $\left\{\chi^{q} \mid q \in Q\right\}$ is a generating set of $k[S]$ as a $k\left[\mathbf{X}_{E}\right]$ module.

Any element in $\Gamma$ whose leading monomial $\mathbf{X}_{E}^{v} \mathbf{X}_{A}^{u}$ has variables in $\left\{X_{i} \mid 1 \leq\right.$ $i \leq f\}$ (i.e. $v \neq 0$ ) is, except $\operatorname{sign} \pm$,

$$
\mathbf{X}_{E}^{v} \mathbf{X}_{A}^{u}-\mathbf{X}_{E}^{v^{\prime}} \mathbf{X}_{A}^{u^{\prime}}
$$


Property 2 of the order says that $v^{\prime} \neq 0$, and therefore, since $\Gamma$ is a reduced Gröbner basis, $\mathbf{X}_{A}^{u}$ and $\mathbf{X}_{A}^{u^{\prime}} \in \mathcal{B}$. Moreover, $u \neq u^{\prime}$ because otherwise, since $I$ is a saturated ideal, $\mathbf{X}_{E}^{v}-\mathbf{X}_{E}^{v^{\prime}} \in I$, which is a contradiction with $\Gamma$ a Gröbner basis.

Suppose that $\mathbf{X}_{A}^{u}=\mathbf{X}_{A}^{\alpha_{i}}$ and $\mathbf{X}_{A}^{u^{\prime}}=\mathbf{X}_{A}^{\alpha_{j}}$.

For any $\gamma \in \mathbb{N}^{r}$ such that

$$
\mathbf{X}_{A}^{\gamma+\alpha_{i}} \in \mathcal{B} \quad \text { and } \quad \mathbf{X}_{A}^{\gamma+\alpha_{j}} \in \mathcal{B},
$$

if $\alpha_{i^{\prime}}=\gamma+\alpha_{i}$ and $\alpha_{j^{\prime}}=\gamma+\alpha_{j}$, we associate with the chosen element in $\Gamma$, the element in $k\left[\mathbf{X}_{E}\right]^{l_{0}}$ with all the coordinates equal to zero, except the $i^{\prime}$ th and $j^{\prime}$ th ones, which are $\mathbf{X}_{E}^{v}$, and $-\mathbf{X}_{E}^{v^{\prime}}$, respectively. Notice that there is at least an element associated, the one corresponding to $\gamma=0$.

For any $\gamma \in \mathbb{N}^{r}$ such that

$$
\mathbf{X}_{A}^{\gamma+\alpha_{i}} \in \mathcal{B} \quad \text { and } \quad \mathbf{X}_{A}^{\gamma+\alpha_{j}} \notin \mathcal{B}
$$

if $\alpha_{i^{\prime}}=\gamma+\alpha_{i}$ and $\mathbf{X}_{E}^{w} \mathbf{X}_{A}^{\alpha_{j^{\prime}}}$ is the division remainder of $\mathbf{X}_{A}^{\gamma+\alpha_{j}}$ by $\Gamma$, we associate with the chosen element in $\Gamma$, the element in $k\left[\mathbf{X}_{E}\right]^{l_{0}}$ with all the coordinates equal to zero, except the $i^{\prime}$ th and $j^{\prime}$ th ones, which are $\mathbf{X}_{E}^{v}$, and $-\mathbf{X}_{E}^{v^{\prime}+w}$, respectively.

In this way, taking all the elements in $\Gamma$ of the above form, we obtain $G_{i} \in$ $k\left[\mathbf{X}_{E}\right]^{l_{0}}, 1 \leq i \leq l_{1}$. Let $\mathcal{N}$ be the matrix

$$
\mathcal{N}=\left(G_{1}|\ldots| G_{l_{1}}\right)
$$

$\mathcal{N}$ defines a morphism of free $k\left[\mathbf{X}_{E}\right]$-modules

$$
\Psi_{1}: k\left[\mathbf{X}_{E}\right]^{l_{1}} \longrightarrow k\left[\mathbf{X}_{E}\right]^{l_{0}} .
$$

Proposition 1.3.

$$
\operatorname{coker}(\mathcal{N}) \simeq_{k\left[\mathbf{X}_{E}\right]} k[S] .
$$

Proof. Since $\operatorname{coker}(\mathcal{N}) \simeq k\left[\mathbf{X}_{E}\right]^{l_{0}} / i m \Psi_{1}$ and $k[S] \simeq k\left[\mathbf{X}_{E}\right]^{l_{0}} / k e r \Psi_{0}$, it is enough to prove that

$$
i m \Psi_{1}=k e r \Psi_{0} .
$$

It is clear that $i m \Psi_{1} \subset \operatorname{ker} \Psi_{0}$. Let $\left(F_{1}\left(\mathbf{X}_{E}\right), \ldots, F_{l_{0}}\left(\mathbf{X}_{E}\right)\right) \in k e r \Psi_{0}$ be a homogeneous element of degree $m \in S$. This means that if $F_{i} \neq 0$, then $F_{i}$ is homogeneous of degree $m-q_{i}$, where $q_{i}$ is the $S$-degree of $\mathbf{X}_{A}^{\alpha_{i}}$. The element $F=\sum_{i=1}^{l_{0}} F_{i} \mathbf{X}_{A}^{\alpha_{i}} \in I$, and therefore the remainder of the Hironaka division of $F$ by $\Gamma$ is 0 .

Notice that if $F_{i} \neq 0$, then $F_{i} \notin k$; otherwise, $\mathbf{X}_{A}^{\alpha_{i}}$ would appear in the remainder. Thus, $F=0$ and we are done, or the leading monomial of $F$ is $\pm \mathbf{X}_{E}^{\omega} \mathbf{X}_{A}^{\alpha_{i}}$ for some $i$, and $\omega \neq 0$. In the latter case, there exists

$$
\pm\left(\mathbf{X}_{E}^{v} \mathbf{X}_{A}^{\alpha_{i^{\prime}}}-\mathbf{X}_{E}^{v^{\prime}} \mathbf{X}_{A}^{\alpha_{j^{\prime}}}\right) \in \Gamma
$$

with leading monomial $\mathbf{X}_{E}^{v} \mathbf{X}_{A}^{\alpha_{i^{\prime}}}, \alpha_{i}=\alpha_{i^{\prime}}+\gamma, \omega=v+\beta$, and $v \neq 0$. Assume, for simplicity's sake, that both elements are positive.

If $\mathbf{X}_{A}^{\alpha_{j^{\prime}}+\gamma}=\mathbf{X}_{A}^{\alpha_{j}} \in \mathcal{B}$, we consider

$$
F^{(1)}:=F-\mathbf{X}_{E}^{\beta}\left(\mathbf{X}_{E}^{v} \mathbf{X}_{A}^{\alpha_{i}}-\mathbf{X}_{E}^{v^{\prime}} \mathbf{X}_{A}^{\alpha_{j}}\right) \in I .
$$

We can write

$$
F^{(1)}=\sum_{l=1}^{l_{0}} F_{l}^{(1)} \mathbf{X}_{A}^{\alpha_{l}},
$$


where the following equations are satisfied:

$$
\begin{aligned}
& F_{i}^{(1)}=F_{i}-\mathbf{X}_{E}^{\omega}, \\
& F_{j}^{(1)}=F_{j}+\mathbf{X}_{E}^{\beta+v^{\prime}}, \\
& F_{l}^{(1)}=F_{l}, \text { for all } l \neq i, j .
\end{aligned}
$$

Equivalently, if we suppose $i<j$ and denote

$$
G^{(1)}:=(0, \ldots, 0, \underbrace{\mathbf{X}_{E}^{v}}_{i}, 0, \ldots, 0, \underbrace{-\mathbf{X}_{E}^{v^{\prime}}}_{j}, 0, \ldots, 0) \in\left\{G_{1}, \ldots, G_{l_{1}}\right\},
$$

we obtain

$$
\left(F_{1}^{(1)}, \ldots, F_{l_{0}}^{(1)}\right)=\left(F_{1}, \ldots, F_{l_{0}}\right)-\mathbf{X}_{E}^{\beta} G^{(1)} .
$$

If $\mathbf{X}_{A}^{\alpha_{j^{\prime}}+\gamma} \notin \mathcal{B}$, let $\mathbf{X}_{E}^{w^{\prime}} \mathbf{X}_{A}^{\alpha_{j}}$ be the division remainder of $\mathbf{X}_{A}^{\alpha_{j^{\prime}}+\gamma}$ by $\Gamma$. We consider

$$
F^{(1)}:=F-\mathbf{X}_{E}^{\beta}\left(\mathbf{X}_{E}^{v} \mathbf{X}_{A}^{\alpha_{i}}-\mathbf{X}_{E}^{w^{\prime}+v^{\prime}} \mathbf{X}_{A}^{\alpha_{j}}\right)=\sum_{l=1}^{l_{0}} F_{l}^{(1)} \mathbf{X}_{A}^{\alpha_{l}} \in I
$$

Now,

$$
\left(F_{1}^{(1)}, \ldots, F_{l_{0}}^{(1)}\right)=\left(F_{1}, \ldots, F_{l_{0}}\right)-\mathbf{X}_{E}^{\beta} G^{(1)},
$$

where if we suppose $i<j$

$$
G^{(1)}:=(0, \ldots, 0, \underbrace{\mathbf{X}_{E}^{v}}_{i}, 0, \ldots, 0, \underbrace{-\mathbf{X}_{E}^{w^{\prime}+v^{\prime}}}_{j}, 0, \ldots, 0) \in\left\{G_{1}, \ldots, G_{l_{1}}\right\} .
$$

In any case, if $F^{(1)}=0$, then $F_{l}^{(1)}=0$ for any $l$, and we are done.

If $F^{(1)} \neq 0$, notice that the leading monomial of $F^{(1)}$ is less than the leading monomial of $F$. We can proceed by recurrence.

Suppose that for fixed $r \geq 2$ and for any $j, 1 \leq j \leq r-1$, there exists

$$
F^{(j)}=F_{1}^{(j)}\left(\mathbf{X}_{E}\right) \mathbf{X}_{A}^{\alpha_{1}}+\cdots+F_{l_{0}}^{(j)}\left(\mathbf{X}_{E}\right) \mathbf{X}_{A}^{\alpha_{l_{0}}} \in I-\{0\}
$$

satisfying

$$
\left(F_{1}^{(j)}, \ldots, F_{l_{0}}^{(j)}\right)=\left(F_{1}^{(j-1)}, \ldots, F_{l_{0}}^{(j-1)}\right)-\mathbf{X}_{E}^{\beta^{(j)}} G^{(j)} \quad\left(F_{i}^{(0)}=F_{i}\right),
$$

where $G^{(j)} \in\left\{G_{1}, \ldots, G_{l_{1}}\right\}$, and $\beta^{(j)} \in \mathbf{N}^{n} \quad\left(\beta^{(1)}=\beta\right)$.

Reasoning as before, we obtain $G^{(r)} \in\left\{G_{1}, \ldots, G_{l_{1}}\right\}$ and $\mathbf{X}_{E}^{\beta^{(r)}}$ such that if

$$
\left(F_{1}^{(r)}, \ldots, F_{l_{0}}^{(r)}\right)=\left(F_{1}^{(r-1)}, \ldots, F_{l_{0}}^{(r-1)}\right)-\mathbf{X}_{E}^{\beta^{(r)}} G^{(r)},
$$

then

$$
F^{(r)}=\sum_{l=1}^{l_{0}} F_{l}^{(r)} \mathbf{X}_{A}^{\alpha_{l}} \in I
$$

and the leading monomial of $F^{(r)}$ is less than the leading monomial of $F^{(r-1)}$.

Therefore, if $F^{(r)}=0$, we obtain

$$
\left(F_{1}^{(r-1)}, \ldots, F_{l_{0}}^{(r-1)}\right)=\mathbf{X}_{E}^{\beta^{(r)}} G^{(r)}
$$


From

$$
\left(F_{1}^{(r-1)}, \ldots, F_{l_{0}}^{(r-1)}\right)=\left(F_{1}, \ldots, F_{l_{0}}\right)-\sum_{i=1}^{r-2} \mathbf{X}_{E}^{\beta^{(i)}} G^{(i)}
$$

we obtain

$$
\left(F_{1}, \ldots, F_{l_{0}}\right)=\sum_{i=1}^{r-1} \mathbf{X}_{E}^{\beta^{(i)}} G^{(i)}
$$

and we are done.

If $F^{(r)} \neq 0$ the result follows by recurrence, because the elements $F^{(j)}$ are homogeneous of degree $m$, and in each step the leading monomial decreases.

Therefore, we obtain the first step of a free resolution of $k[S]$ as a $k\left[\mathbf{X}_{E}\right]$-module that is $S$-graded:

$$
k\left[\mathbf{X}_{E}\right]^{l_{1}} \stackrel{\Psi_{1}}{\rightarrow} k\left[\mathbf{X}_{E}\right]^{l_{0}} \stackrel{\Psi_{0}}{\rightarrow} k[S] \rightarrow 0 .
$$

In the above proof, for any element $\mathbf{X}_{A}^{\alpha_{i}} \in \mathcal{B}$ we have considered its $S$-degree $q_{i} \in Q$. Assume, for simplicity's sake, that $Q=\left\{q_{1}, \ldots, q_{\beta_{0}}\right\}$, where $\beta_{0}=\sharp Q$. Notice that $\beta_{0} \leq l_{0}$. In the case $\beta_{0}<l_{0}$, if $\beta_{0}+1 \leq i \leq l_{0}, q_{i}=q_{j}$ for a unique $j$, $1 \leq j \leq \beta_{0}$. Denote $j=j(i)$. We consider

$$
\pi: k\left[\mathbf{X}_{E}\right]^{l_{0}} \rightarrow k\left[\mathbf{X}_{E}\right]^{\beta_{0}},
$$

the $k\left[\mathbf{X}_{E}\right]$-module morphism defined by

$$
\pi\left(e_{i}\right)= \begin{cases}e_{i} & \text { if } 1 \leq i \leq \beta_{0} \\ e_{j(i)} & \text { if } \beta_{0}+1 \leq i \leq l_{0} .\end{cases}
$$

Notice that $\pi \circ \Psi_{1}: k\left[\mathbf{X}_{E}\right]^{l_{1}} \rightarrow k\left[\mathbf{X}_{E}\right]^{\beta_{0}}$ is given by the matrix

$$
\mathcal{M}:=\left(\pi\left(G_{1}\right)|\ldots| \pi\left(G_{l_{1}}\right)\right) .
$$

On the other hand, considering the morphism of $k\left[\mathbf{X}_{E}\right]$-modules

$$
\Phi_{0}: k\left[\mathbf{X}_{E}\right]^{\beta_{0}} \longrightarrow k[S]
$$

defined by $\Phi_{0}\left(e_{i}\right)=\chi^{q_{i}}, 1 \leq i \leq \beta_{0}$. It is clear that $\Phi_{0} \circ \pi=\Psi_{0}$.

\section{Proposition 1.4.}

$$
\operatorname{coker}(\mathcal{M}) \simeq_{k\left[\mathbf{x}_{E}\right]} k[S] .
$$

Proof. The situation is the following:

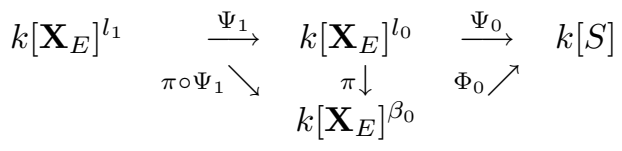

As in Proposition 1.3, it is enough to prove that

$$
\operatorname{im}\left(\pi \circ \Psi_{1}\right)=\operatorname{ker}\left(\Phi_{0}\right) .
$$

$i m\left(\pi \circ \Psi_{1}\right) \subset \operatorname{ker}\left(\Phi_{0}\right)$ follows from $\Phi_{0}\left(\pi\left(G_{i}\right)\right)=\Psi_{0}\left(G_{i}\right)=0$, for any $i, 1 \leq i \leq l_{1}$.

For the other inclusion, let $\left(F_{1}\left(\mathbf{X}_{E}\right), \ldots, F_{\beta_{0}}\left(\mathbf{X}_{E}\right)\right) \in \operatorname{ker} \Phi_{0}$. Thus,

$$
\left(F_{1}\left(\mathbf{X}_{E}\right), \ldots, F_{\beta_{0}}\left(\mathbf{X}_{E}\right), 0, \ldots, 0\right) \in \operatorname{ker} \Psi_{0}=i m \Psi_{1} \text {. }
$$


There exist $\lambda_{i}\left(\mathbf{X}_{E}\right)$ such that $\left(F_{1}, \ldots, F_{\beta_{0}}, 0, \ldots, 0\right)=\sum_{i=1}^{l_{1}} \lambda_{i} G_{i}$. Notice that if we denote $G_{i j}$ the $j$ th coordinate of $G_{i}$, then

$$
\sum_{i=1}^{l_{1}} \lambda_{i} G_{i j}=0
$$

for any $j, \beta_{0}+1 \leq j \leq l_{0}$. Therefore, if we denote $\pi\left(G_{i}\right)_{t}$ the $t$ th coordinate of $\pi\left(G_{i}\right)$, notice that

$$
\pi\left(G_{i}\right)_{t}=G_{i t}+\sum_{j(s)=t, \beta_{0}+1 \leq s \leq l_{0}} G_{i s}, \quad 1 \leq t \leq \beta_{0} .
$$

Thus, for a fixed $t$

$$
\sum_{i=1}^{l_{1}} \lambda_{i} \pi\left(G_{i}\right)_{t}=\sum_{i=1}^{l_{1}} \lambda_{i} G_{i t}
$$

Therefore,

$$
\left(F_{1}, \ldots, F_{\beta_{0}}\right)=\sum_{i=1}^{l_{1}} \lambda_{i} \pi\left(G_{i}\right)
$$

and we are done.

From the free resolution

$$
k\left[\mathbf{X}_{E}\right]^{l_{1}} \stackrel{\pi \circ \Psi_{1}}{\rightarrow} k\left[\mathbf{X}_{E}\right]^{\beta_{0}} \stackrel{\Phi_{0}}{\rightarrow} k[S] \rightarrow 0,
$$

using the Schreyer Theorem and its improvements (see [11]), the $S$-graded minimal free resolution of $k[S]$ as a $k\left[\mathbf{X}_{E}\right]$-module can be obtained. We will call this resolution the short resolution of $k[S]$ to distinguish it from the minimal free resolution of $k[S]$ as a $k[\mathbf{X}]$-module.

\section{Combinatorial Description of the Short Resolution}

Assume that $S \neq(0)$, and consider the $S$-graded minimal free resolution of $k[S]$ as a $k\left[\mathbf{X}_{E}\right]$-module

$$
0 \rightarrow k\left[\mathbf{X}_{E}\right]^{\beta_{f-1}} \stackrel{\Phi_{f-1}}{\rightarrow} \cdots \rightarrow k\left[\mathbf{X}_{E}\right]^{\beta_{2}} \stackrel{\Phi_{2}}{\rightarrow} k\left[\mathbf{X}_{E}\right]^{\beta_{1}} \stackrel{\Phi_{1}}{\rightarrow} k\left[\mathbf{X}_{E}\right]^{\beta_{0}} \stackrel{\Phi_{0}}{\rightarrow} k[S] \rightarrow 0
$$

The $S$-graded Nakayama's lemma (see [3]) says this resolution is unique except for isomorphisms. Moreover, denoting $M_{i}=\operatorname{ker}\left(\Phi_{i}\right)$ as the $i$ th module of syzygies of $k[S]$ as a $k\left[\mathbf{X}_{E}\right]$-module, $0 \leq i \leq f-1$, we obtain

$$
\beta_{i+1}=\sum_{m \in S} \operatorname{dim} W_{i}(m)
$$

where $W_{i}(m):=\left(M_{i} / \mathfrak{m}_{E} M_{i}\right)_{m}$ is considered as a $k$-vector space, and $\mathfrak{m}_{E}$ is the ideal of $k\left[\mathbf{X}_{E}\right]$ generated by the indeterminates of $\mathbf{X}_{E}\left(X_{i}\right.$ such that $\left.m_{i} \in E\right)$.

We will show how this resolution can be described by means of some simplicial complexes. Concretely, if $m \in S$, let $T_{m}$ be the simplicial complex

$$
T_{m}=\left\{F \subset E \quad \mid m-n_{F} \in S\right\} .
$$

Denote $\widetilde{H}_{i}\left(T_{m}\right)$ the $i$ th reduced homology space of the simplicial complex $T_{m}$, and let $\tilde{h}_{i}\left(T_{m}\right)=\operatorname{dim}\left(\tilde{H}_{i}\left(T_{m}\right)\right)$. 


\section{Proposition 2.1.}

$$
\tilde{H}_{i}\left(T_{m}\right) \simeq W_{i}(m),
$$

for any $m \in S$ and for any $i, 0 \leq i \leq f-2$.

Proof. Let us consider $k[S]$ and $k \simeq k\left[\mathbf{X}_{E}\right] / \mathfrak{m}_{E}$ as $k\left[\mathbf{X}_{E}\right]$-modules and use the commutativity of the functor Tor, concretely

$$
\operatorname{Tor}_{i+1}(k[S], k) \simeq \operatorname{Tor}_{i+1}(k, k[S]) .
$$

In order to compute the space $\operatorname{Tor}_{i+1}(k[S], k)$ as $k\left[\mathbf{X}_{E}\right]$-module, take the Koszul complex for the regular sequence $\left\{X_{i} \mid m_{i} \in E\right\}$, which is an exact sequence.

$$
\begin{aligned}
0 \rightarrow \bigwedge^{f} k\left[\mathbf{X}_{E}\right]^{f} \stackrel{d_{f-1}}{\rightarrow} \cdots \rightarrow \bigwedge^{j+1} k\left[\mathbf{X}_{E}\right]^{f} \stackrel{d_{j}}{\rightarrow} \bigwedge^{j} k\left[\mathbf{X}_{E}\right]^{f} \\
\stackrel{d_{j-1}}{\rightarrow} \cdots \rightarrow k\left[\mathbf{X}_{E}\right]^{f} \stackrel{d_{0}}{\rightarrow} k\left[\mathbf{X}_{E}\right] \rightarrow k \rightarrow 0 .
\end{aligned}
$$

Here $d_{j}$ is given by

$$
d_{j}\left(e_{i_{0}} \wedge \cdots \wedge e_{i_{j}}\right)=\sum_{l=0}^{j}(-1)^{l} X_{l} e_{i_{0}} \wedge \cdots \wedge e_{i_{l-1}} \wedge e_{i_{l+1}} \wedge \cdots \wedge e_{i_{j}} .
$$

These homomorphism are $S$-graded of degree 0 assigning the degree $m_{i_{0}}+\cdots+m_{i_{j}}$ to the element $e_{i_{0}} \wedge \cdots \wedge e_{i_{j}}$. Tensoring this exact sequence with the $k\left[\mathbf{X}_{E}\right]$-module $k[S]$, we obtain the $S$-graded Koszul complex

$$
0 \rightarrow \bigwedge^{f} k[S]^{f} \rightarrow \cdots \rightarrow \bigwedge^{j+1} k[S]^{f} \stackrel{d_{j}}{\rightarrow} \bigwedge^{j} k[S]^{f} \stackrel{d_{j-1}}{\rightarrow} \cdots \rightarrow k[S]^{f} \stackrel{d_{0}}{\rightarrow} k[S] \rightarrow k \rightarrow 0 .
$$

The restriction to its degree $m \in S$ is the following complex of finite-dimensional $k$-vector space

$$
\cdots \rightarrow \bigoplus_{\substack{F \subset E \\ \sharp F=3}} k[S]_{m-n_{F}} \rightarrow \bigoplus_{\substack{F \subset E \\ \sharp F=2}} k[S]_{m-n_{F}} \rightarrow \bigoplus_{\substack{F \subset E \\ \sharp F=1}} k[S]_{m-n_{F}} \rightarrow k[S]_{m} \rightarrow 0 .
$$

Notice that this complex can be identified with the augmented-oriented chain complex of $T_{m}$, because

$$
k[S]_{m-n_{F}}=\left\{\begin{array}{l}
k, \text { if } F \in T_{m} \\
0, \text { otherwise }
\end{array}\right.
$$

Thus, we obtain that

$$
\left(\operatorname{Tor}_{i+1}(k[S], k)\right)_{m} \simeq \widetilde{H}_{i}\left(T_{m}\right) .
$$

In order to compute $\operatorname{Tor}_{i+1}(k, k[S])$ as a $k\left[\mathbf{X}_{E}\right]$-module, take the $S$-graded minimal free resolution of $k[S]$ as a $k\left[\mathbf{X}_{E}\right]$-module. Tensoring with $k \simeq k\left[\mathbf{X}_{E}\right] / \mathfrak{m}_{E}$ it is obtained

$$
\begin{aligned}
0 \rightarrow\left(k\left[\mathbf{X}_{E}\right] / \mathfrak{m}_{E}\right)^{\beta_{f-1}} \stackrel{\tilde{\Phi}_{f-1}}{\longrightarrow} \cdots \rightarrow\left(k\left[\mathbf{X}_{E}\right] / \mathfrak{m}_{E}\right)^{\beta_{2}} & \\
& \stackrel{\tilde{\Phi}_{2}}{\rightarrow}\left(k\left[\mathbf{X}_{E}\right] / \mathfrak{m}_{E}\right)^{\beta_{1}} \stackrel{\tilde{\Phi}_{1}}{\rightarrow}\left(k\left[\mathbf{X}_{E}\right] / \mathfrak{m}_{E}\right)^{\beta_{0}} \rightarrow 0 .
\end{aligned}
$$

Thus, $\left(\operatorname{Tor}_{i+1}(k, k[S])\right)_{m} \simeq W_{i}(m)$.

Now it is clear that the isomorphism follows from the commutativity of the functor Tor. 
As an application of these isomorphisms, if denote

$$
D(i):=\left\{m \in S \quad \mid \widetilde{H}_{i}\left(T_{m}\right) \neq 0\right\},
$$

we obtain that

$$
\beta_{i+1}=\sum_{i \in D(i)} \widetilde{h}_{i}\left(T_{m}\right),-1 \leq i \leq f-2 .
$$

Notice that, by the noetherian property, $D(i)$ is finite. These sets $D(i)$ can be computed from the short resolution. Moreover, we can state the following corollary.

Corollary 2.2. The regularity of a homogeneous lattice ideal I can be computed from the short resolution.

Proof. Assume that $I$ is a homogeneous ideal for the natural grading. In this case, it is well defined that $\|m\|=\|\alpha\|_{1}$, where $m=\sum_{i=1}^{n} \alpha_{i} m_{i}$ and $\|\alpha\|_{1}=\sum_{i=1}^{n} \alpha_{i}$.

It is enough to use the following formula $([\underline{4}])$ :

$$
\operatorname{reg}(I)=\max _{-1 \leq i \leq f-2}\left\{u_{i}-i\right\},
$$

where $u_{i}=\max \{\|m\| \mid m \in D(i)\}$.

Remark 2.3. The results in section 1 allow the computation of the sets $D(i)$ using Gröbner Bases. This method is more useful for explicit computations than that in [4] using Hilbert bases of some diophantine systems (see [10]).

Example 2.4. Consider the semigroup $S \subset \mathbb{N}^{3}$ generated by

$$
[5,0,0],[0,5,0],[0,0,5],[4,1,0],[1,4,0],[2,3,0],[0,1,4],[0,4,1],[0,2,3],
$$

a projective simplicial toric surface.

The Gröbner basis of $I$ with respect to lex-inf is

$$
\begin{aligned}
\Gamma=\{ & -x_{3} x_{8}+x_{9}^{2}, x_{9} x_{8}-x_{2} x_{7}, x_{8}^{3}-x_{2}^{2} x_{9},-x_{2} x_{3}+x_{7} x_{8}, \\
& -x_{9} x_{3}+x_{7}^{2},-x_{2} x_{4}+x_{6}^{2}, x_{5}^{2}-x_{6} x_{2},-x_{1} x_{5}+x_{6} x_{4}, \\
& \left.x_{4} x_{5}-x_{1} x_{2},-x_{1}^{2} x_{6}+x_{4}^{3}, \underline{-x_{2} x_{7} x_{9}+x_{3} x_{8}^{2}}, \underline{x_{2} x_{4}^{2}-x_{6} x_{1} x_{5}}\right\} .
\end{aligned}
$$

Thus,

$$
\begin{aligned}
\mathcal{B}=\{ & 1, x_{4}, x_{4}^{2}, x_{5}, x_{6}, x_{5} x_{6}, x_{7}, x_{4} x_{7}, x_{4}^{2} x_{7}, \\
& x_{5} x_{7}, x_{6} x_{7}, x_{5} x_{6} x_{7}, x_{8}, x_{4} x_{8}, x_{4}^{2} x_{8}, \\
& x_{5} x_{8}, x_{6} x_{8}, x_{5} x_{6} x_{8}, x_{8}^{2}, x_{4} x_{8}^{2}, x_{4}^{2} x_{8}^{2}, x_{5} x_{8}^{2}, \\
& x_{6} x_{8}^{2}, x_{5} x_{6} x_{8}^{2}, x_{9}, x_{4} x_{9}, x_{4}^{2} x_{9}, x_{5} x_{9}, x_{6} x_{9}, x_{5} x_{6} x_{9}, \\
& \left.x_{7} x_{9}, x_{4} x_{7} x_{9}, x_{4}^{2} x_{7} x_{9}, x_{5} x_{7} x_{9}, x_{6} x_{7} x_{9}, x_{5} x_{6} x_{7} x_{9}\right\},
\end{aligned}
$$

and

$$
\begin{aligned}
Q=\{ & {[0,0,0],[4,1,0],[8,2,0],[1,4,0],[2,3,0],[3,7,0],[0,1,4],[4,2,4],[8,3,4], } \\
& {[1,5,4],[2,4,4],[3,8,4],[0,4,1],[4,5,1],[8,6,1],[1,8,1],[2,7,1],[3,11,1], } \\
& {[0,8,2],[4,9,2],[8,10,2],[1,12,2],[2,11,2],[3,15,2],[0,2,3],[4,3,3],[8,4,3], } \\
& {[1,6,3],[2,5,3],[3,9,3],[0,3,7],[4,4,7],[8,5,7],[1,7,7],[2,6,7],[3,10,7]\}, }
\end{aligned}
$$

and therefore $u_{-1}=4$. Notice that $\sharp \mathcal{B}=\sharp Q=36$ and thus $\Phi_{0}=\Psi_{0}$. 
In order to construct the matrix $\mathcal{N}$, from the underlined binomial in $\Gamma$ we obtain twelve elements $G_{i} \in \operatorname{ker}\left(\Psi_{0}\right)$ and $u_{0}=5$.

Notice that if we denote $\alpha_{1}=X_{4}^{2} X_{8}^{2}, \alpha_{2}=X_{4}^{2} X_{7} X_{9}, \alpha_{3}=X_{5} X_{6} X_{8}^{2}$ and $\alpha_{4}=X_{5} X_{6} X_{7} X_{9}$, the elements

$$
\begin{aligned}
& G_{1}=\left(X_{3},-X_{2}, 0, \ldots, 0\right), \\
& G_{2}=\left(X_{2}, 0,-X_{1}, 0, \ldots, 0\right), \\
& G_{3}=\left(0, X_{2}, 0,-X_{1}, 0, \ldots, 0\right), \\
& G_{4}=\left(0,0, X_{3},-X_{2}, 0, \ldots, 0\right),
\end{aligned}
$$

are the unique ones with some non-null coordinates in some common position.

It is easy to see that the unique possible minimal relation between them is

$$
X_{2} G_{1}-X_{3} G_{2}+X_{2} G_{3}-X_{1} G_{4}=0 .
$$

Therefore, $u_{1}=6$.

The short resolution is

$$
0 \rightarrow k\left[\mathbf{X}_{E}\right] \stackrel{\Phi_{2}}{\rightarrow} k\left[\mathbf{X}_{E}\right]^{12} \stackrel{\Phi_{1}}{\rightarrow} k\left[\mathbf{X}_{E}\right]^{36} \stackrel{\Phi_{0}}{\rightarrow} k[S] \rightarrow 0 .
$$

The regularity of $I$ is

$$
\operatorname{reg}(I)=\max \left\{u_{1}-1=5, u_{0}-0=5, u_{-1}+1=5\right\}=5 .
$$

\section{ACKNOWLEDGEMENT}

The author thanks Bernd Sturmfels for his suggestions about the results in this paper during a visit to Berkeley in the summer of 2000.

\section{REFERENCES}

[1] R. APERY, Sur les branches superlinéaires des courbes algébriques. C.R. Acad. Sci. Paris, 222, (1946), 1198-1200. MR 8:221a

[2] D. BAYER, B. STURMFELS, Cellular resolutions of monomial modules. J. reine angew. Math., 502, (1998), 123-140. MR 99g:13018

[3] E. BRIALES, A. CAMPILlO, C. MARIJUÁN, P. PISÓN, Minimal Systems of Generators for Ideals of Semigroups. J. of Pure and Applied Algebra, 124, (1998), 7-30. MR 98k:20105

[4] E. BRIAlES, A. CAMPILlO, P. PISÓN, A. VIGNERON, Simplicial Complexes and Syzygies of Lattice Ideals. in Symbolic Computation: Solving Equations in Algebra, Geometry and Engineering, American Mathematical Society, Contemporary Mathematics, 286, (2001), 169183.

[5] E. BRIALES, P. PISÓN, A. VIGNERON, The Regularity of a Toric Variety. Journal of Algebra, 237, (2001), 165-185.

[6] A. CAMPILlO, P. GIMÉNEZ, Syzygies of affine toric varieties. Journal of Algebra, 225, (2000), 142-161. MR 2001j:13014

[7] A. CAMPILlO, C. MARIJUÁN, Higher relations for a numerical semigroup. Sém. Théor. Nombres Bordeaux, 3, (1991), 249-260.

[8] L. GRUSON, R. LAZARSFELD, C. PESKINE, On a theorem of Castelnuovo and equations defining space curves. Invent. Math., 72, (1983), 491-506. MR 85g:14033

[9] S. L'VOVSKY, On inflection points, monomial curves, and hypersurfaces containing projective curves. Math. Ann., 306, (1996), 719-735. MR 99e:14033

[10] P. PISÓN-CASARES, A. VIGNERON-TENORIO, First Syzygies of Toric Varieties and Diophantine Equations in Congruence. Communications in Algebra, 29, 4, (2001).

[11] R. LA SCALA, M. STILLMAN, Strategies for Computing Minimal Free Resolutions, J. Symbolic Computation, 26, (1998), 409-431. MR 99k:13039 
[12] B. STURMFELS, Gröbner Bases and Convex Polytopes. AMS University Lectures Series, Vol. 8 (1996). MR 97b:13034

[13] A. VIGNERON-TENORIO, Semigroup Ideals and Linear Diophantine Equations. Linear Algebra and its Applications, 295 (1999), 133-144.

Departamento de Álgebra, Facultad de Matemáticas, University of Sevilla, Apartado 1160, 41080 Sevilla, Spain

E-mail address: pilar@algebra.us.es

E-mail address: ppison@us.es 